\title{
双官能团柱[5]芳烃的合成
}

\author{
苏戈吴斗峰李巧伟侯军利* \\ (复旦大学化学系 上海 200433)
}

\begin{abstract}
摘要 以柱 [5]芳烃为原料, 发展了一种由氨基与酯基以及氨基和羧基双官能团衍生化柱[5]芳烃的合成方法, 并通过 ${ }^{1} \mathrm{H}$ NMR 以及 X 射线单晶衍射实验对柱[5]芳烃中官能团的构象进行了研究. 在这类柱[5]芳烃中, 分子内氢键可以诱导 骨架上的侧链发生内翻, 使得侧链上的氨基位于柱 [5]芳烃孔穴内, 并且通过调节烷基链的长度可以控制氨基的位置.

关键词 柱[5]芳烃; 双官能团化; 合成; 构象分析
\end{abstract}

\section{Synthesis of the Bi-functionalized Pillar[5]arene}

\author{
$\mathrm{Su}, \mathrm{Ge} \quad \mathrm{Wu}$, Doufeng Li, Qiaowei Hou, Junli* \\ ( ${ }^{a}$ Department of Chemistry, Fudan University, Shanghai 200433)
}

\begin{abstract}
The method for the synthesis of bi-functionalized pillar[5]arenes with amino and ester groups or amino and carboxylic group has been developed. The conformation of the functional group of the pillar[5]arene was investigated by using ${ }^{1} \mathrm{H}$ NMR and X-Ray experiments. The intramolecular hydrogen bonding induces the side-chain of the pillar[5]arene to introvert. And thus, the amino group points to the inside cavity of the pillar[5] arene backbone. The position of the amino group is adjustable by using alkyl chains with different length.

Keywords pillar[5]arene; bi-functionalize; synthesis; conformation analysis
\end{abstract}

作为一类新型的大环化合物，柱[5]芳烃在其合成 之初就受到了超分子化学家的重视 ${ }^{[1]}$. 由于柱[5]芳烃具 有规则的柱状空腔结构, 并展现出了优良的分子识别性 质，被认为是继冠醚、环糊精、杯芳烃、葫芦脲之后新 的一代大环主体化合物 ${ }^{[2]}$. 另外，由于柱[5]芳烃还具有 广泛的可修饰性 ${ }^{[3]}$, 也为其在超分子聚合物 ${ }^{[4]}$ 、分子器 件 ${ }^{[5]}$ 、纳米通道 ${ }^{[6]}$ 、生物医药 ${ }^{[7]}$ 、功能材料 ${ }^{[8]}$ 等领域的应 用提供了可能. 目前, 柱[5]芳烃的修饰方法主要有全修 饰 ${ }^{[9]}$ 、半修饰 ${ }^{[10]}$ 和单修饰 ${ }^{[1]}$. 基于这些方法, 已经分别 发展出了带 10 个和 5 个相同官能团及单个官能团的柱 [5]芳烃 ${ }^{[12]}$. 但是, 在柱 [5]芳烃骨架上引入多种官能团 还存在较大的困难. 在本工作中, 我们发展了一种构筑 双官能团柱 [5]芳烃的方法, 合成了由氨基与酯基以及 氨基和羧基双官能团衍生化的柱[5]芳烃, 并对这类柱 [5]芳烃的官能团进行了构象分析.

\section{1 结果与讨论}

\section{1 双官能团柱[5]芳烃的合成}

我们以柱[5]芳烃 1 为原料 ${ }^{[1]}$, 参照文献[13, 14]方 法, 经 $\left(\mathrm{NH}_{4}\right)_{2} \mathrm{Ce}\left(\mathrm{NO}_{3}\right)_{6}$ 氧化合成得到了苯醌并柱 [5]芳烃 2, 再经 $\mathrm{NaBH}_{4}$ 还原, 可以得到双羟基衍生化的柱[5]芳 烃 3. 该化合物再与溴乙酸乙酯反应可以 $58 \%$ 的产率得 到双酯基衍生化的化合物 $\mathbf{4}$. 化合物 $\mathbf{4}$ 再与 1.0 equiv.的 $\mathrm{NaOH}$ 反应可以 $28 \%$ 的产率得到单水解产物 $\mathbf{5}$. 单羒基 衍生化的化合物 $\mathbf{5}$ 转变成酰氯后, 可以与单 Boc 保护的 丙二胺反应生成化合物 6. 化合物 6 经三氟乙酸(TFA) 脱除 Boc 保护基后可以得到由氨基和酯基衍生化的柱 [5]芳烃 7 (Scheme 1).

\section{2 双官能团柱[5]芳烃构象研究}

在合成得到双官能团柱[5]芳烃 $7 \mathbf{a} \sim 7 \mathbf{c}$ 后，利用 ${ }^{1} \mathrm{H}$

\footnotetext{
*E-mail: houj1@fudan.edu.cn

Received April 19, 2016; revised May 13, 2016; published online May 19, 2016.

Project supported by the National Natural Science Foundation of China (Nos. 21422202, 21572035).

国家自然科学基金(Nos. 21422202, 21572035)资助项目.
} 


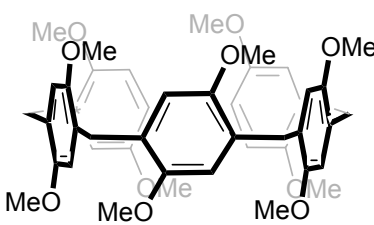

1

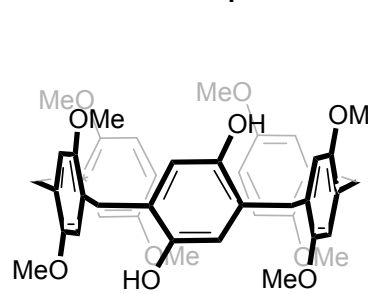

3

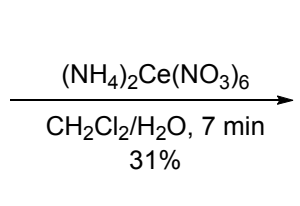

MeO

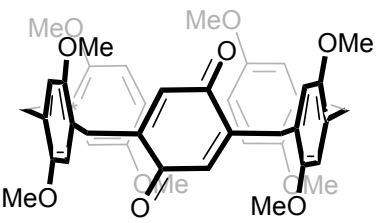

2
$\underset{\mathrm{THF} / \mathrm{CH}_{3} \mathrm{OH}(V: V=6: 1)}{\mathrm{NaBH}_{4}}$

r.t., $0.5 \mathrm{~h}, 83 \%$

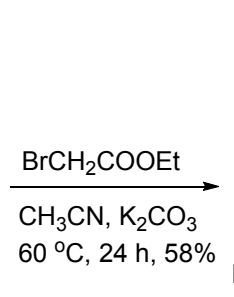
$60{ }^{\circ} \mathrm{C}, 24 \mathrm{~h}, 58 \%$

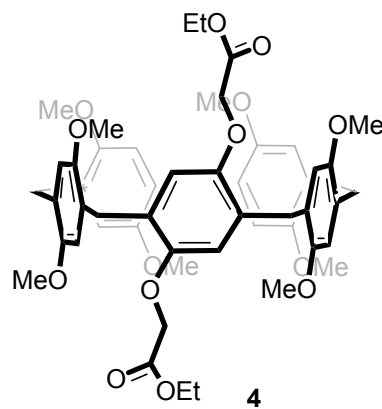

$\stackrel{\mathrm{NaOH}}{\mathrm{THF} / \mathrm{EtOH} / \mathrm{H}_{2} \mathrm{O}}$
$40^{\circ} \mathrm{C}, 1 \mathrm{~h}, 28 \%$
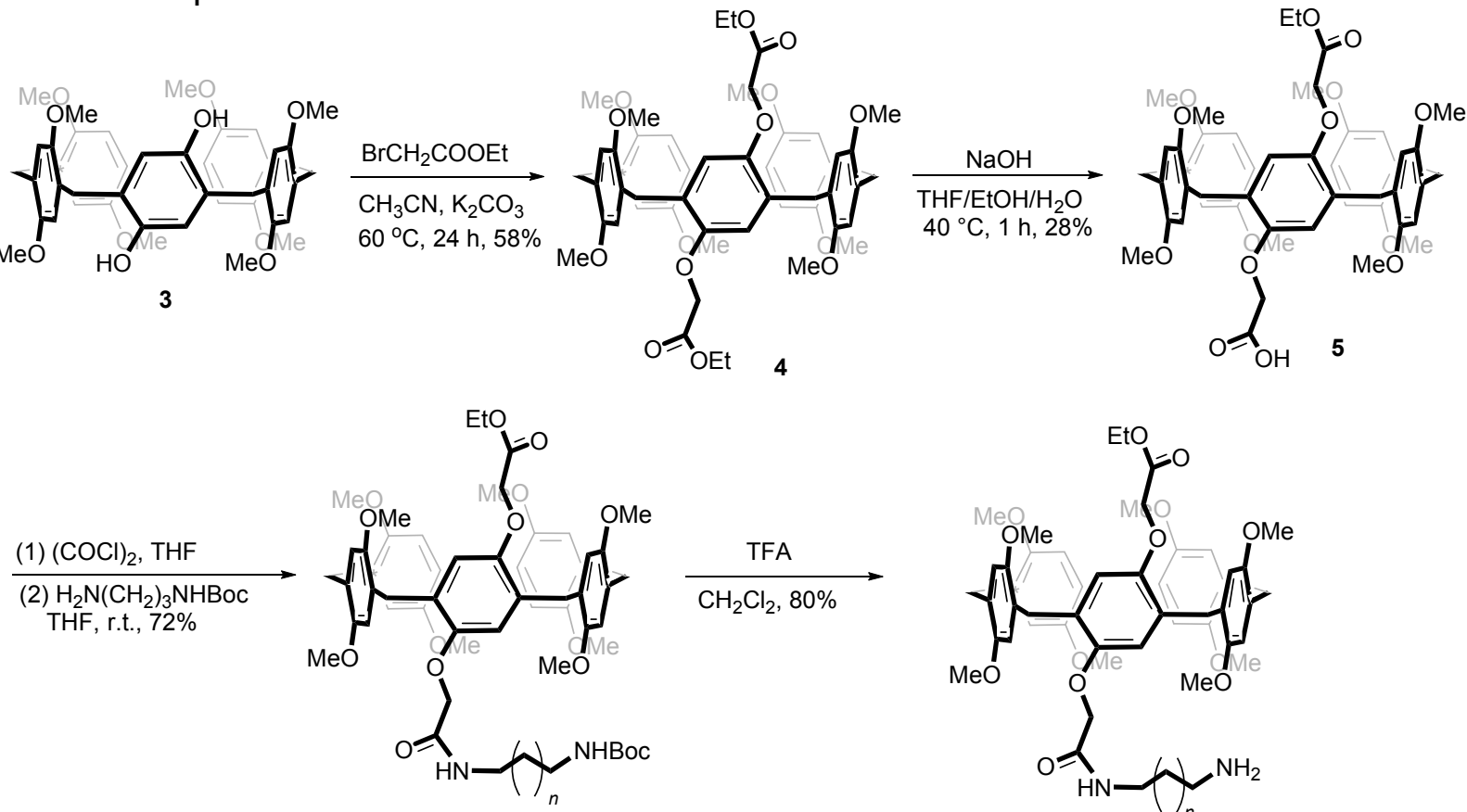

6a: $n=1$

6b: $n=2$

6c: $n=3$

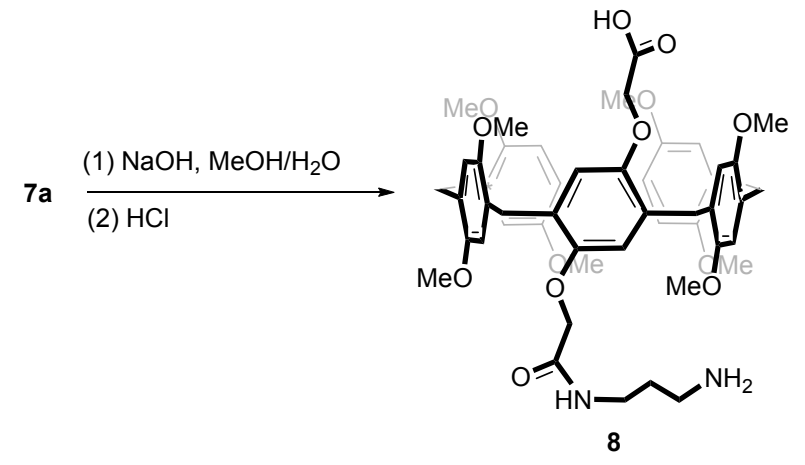

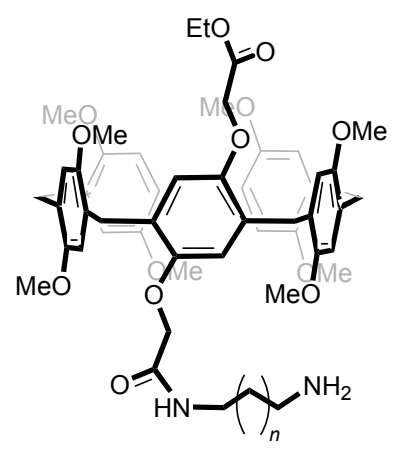

7a: $n=1$

7b: $n=2$

7c: $n=3$

图式 1 化合物 $7 \mathrm{a} \sim 7 \mathrm{c}$ 和 8 的合成方法

Scheme 1 Synthetic method for compounds $7 \mathbf{a} \sim 7 \mathbf{c}$ and 8

NMR 实验对柱 [5]芳烃中氨基的构象进行了研究. 发现 化合物 7a 7 $\mathrm{c}$ 中与氨基相连烷基侧链上的质子信号均 向高场发生了不同程度的移动(图 1), 说明这些侧链发 生了内翻, 使得侧链上的质子位于柱[5]芳烃孔穴内. 由 于柱[5]芳烃骨架上苯环的屏蔽作用, 这些质子的化学 位移均向高场移动, 与苯环距离越近, 质子化学位移越 大. 在前期研究工作中我们发现, 酰胺 $\mathrm{N}-\mathrm{H}$ 可以与柱 [5]芳烃骨架上的氧原子形成分子内的氢键, 从而诱导 侧链发生内翻, 形成自包结的结构 ${ }^{[15]}$. 在化合物 $7 \mathbf{a} \sim 7 \mathbf{c}$ 中, 同样含有可以形成分子内氢键的结构单元, 导致这
类化合物中的侧链发生内翻，使得这类化合物中的氨基 位于柱[5]芳烃孔穴内. 在 $\mathrm{CDCl}_{3}$ 中, 化合物 7a $\sim \mathbf{7}$ 上与 氨基相连亚甲基质子 $\mathrm{H}^{\mathrm{a}}$ 的化学位移分别是 $\delta-0.85,-$ 2.39 和 -2.23 , 说明化合物 $7 \mathbf{b}$ 中 $\mathrm{H}^{\mathrm{a}}$ 最靠近柱 [5]芳烃的 骨架. $\mathrm{C}-\mathrm{N}$ 键与 $\mathrm{C}-\mathrm{C}$ 键具有相似的键长, 可以认为化 合物 7a 中的氨基处于柱 [5]芳烃孔穴的中心. 化合物 $7 \mathrm{a} \sim 7 \mathrm{c}$ 上氨基在孔穴中所处的位置不同说明，通过控 制侧链的长度可以调控氨基在孔穴中的位置. 另外, 化 合物 7a 侧链上与酰胺 $\mathrm{NH}$ 相连亚甲基上的质子 $\mathrm{H}^{\mathrm{c}}$ 为一 个宽峰(图 1a), 说明该侧链构象变化处于 NMR 仪器的 
时间分辨范围之外, 而化合物 $7 \mathbf{b}$ 和 7c 中同样位置亚甲 基上的质子 $\mathrm{H}^{\mathrm{d}}$ 和 $\mathrm{H}^{\mathrm{e}}$ 发生了明显的裂分(图 $1 \mathrm{~b}$ 和 $1 \mathrm{c}$ ), 说 明这两个化合物中侧链的构象稳定, 从而引起亚甲基上 的两个质子构象不同.

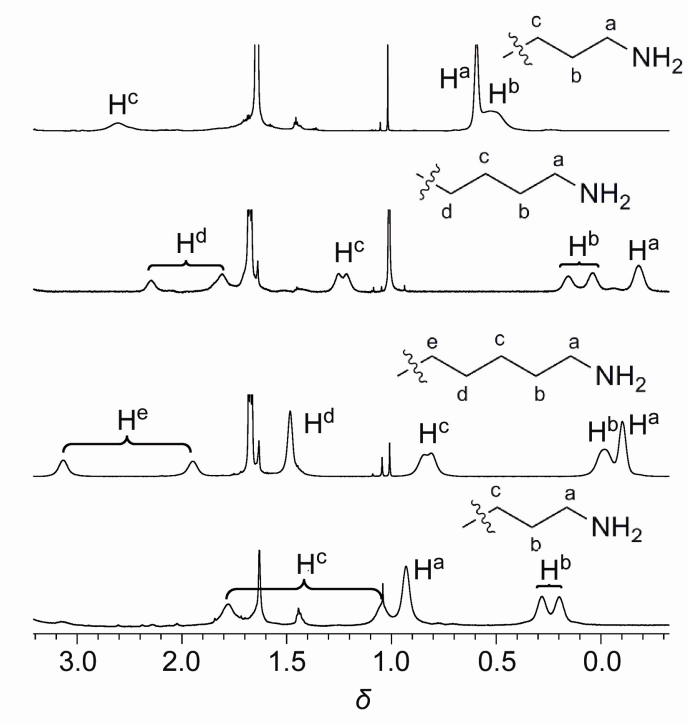

图 1 化合物 7a (a), 7b (b), 7c (c) 和 8 (d) 的部分核磁共振氢谱 $\left(400 \mathrm{MHz}, \mathrm{CDCl}_{3}, 298 \mathrm{~K}, 2.0 \mathrm{mmol} \cdot \mathrm{L}^{-1}\right)$

Figure 1 Partial ${ }^{1} \mathrm{H}$ NMR spectra $\left(400 \mathrm{MHz}, \mathrm{CDCl}_{3}, 298 \mathrm{~K}, 2.0\right.$ $\mathrm{mmol} \cdot \mathrm{L}^{-1}$ ) of $7 \mathbf{a}(\mathrm{a}), 7 \mathbf{b}(\mathrm{b}), 7 \mathbf{c}(\mathrm{c})$ and $\mathbf{8}(\mathrm{d})$

为了进一步合成双官能团的柱[5]芳烃, 我们对化 合物 7a 进行了水解并得了由氨基和羧基双官能团衍生 化的柱[5]芳烃 8 (Scheme 1). 通过 ${ }^{1} \mathrm{H}$ NMR 实验发现, 该化合物中与氨基相连烷基侧链上的质子信号也向高 场发生了移动(图 1d), 说明该侧链也发生了内翻, 使得 氨基位于柱[5]芳烃孔穴内. 与化合物 7a 相比, 化合物 $\mathbf{8}$ 中 $\mathrm{H}^{\mathrm{b}}$ 和 $\mathrm{H}^{\mathrm{c}}$ 的信号明显向高场发生了移动, 并且发生了 明显的裂分, 这主要是由于在溶液相中, 化合物 8 中的 氨基与羧基形成内盐, 从而产生静电吸引作用, 使得烷 基侧链更伸向孔穴的内部, 并且烷基在孔穴中的构象更 加稳定. 通过缓慢挥发的方法, 在 $\mathrm{CH}_{2} \mathrm{Cl}_{2}$ 溶液中得到了 化合物 8 的单晶(图 2). 通过 X 射线晶体结构分析发现, 在分子内氢键作用的诱导下, 烷基侧链发生内翻, 使得 氨基位于柱 [5]芳烃孔穴内. 并且由于铵离子与羧基负 离子的静电相互作用, 另一侧链 $\mathrm{CH}_{2} \mathrm{COO}^{-}$也发生了内 翻, 使得羧基负离子位于孔穴的上方.

\section{2 结论}

以柱[5]芳烃为原料, 通过简单的反应合成了一系 列由氨基与酯基以及氨基和羧基双官能团衍生化的柱 [5]芳烃, 并通过 ${ }^{1} \mathrm{H}$ NMR 以及 X 射线单晶衍射实验对柱 [5]芳烃中的官能团进行了研究. 在这类柱 [5]芳烃中,

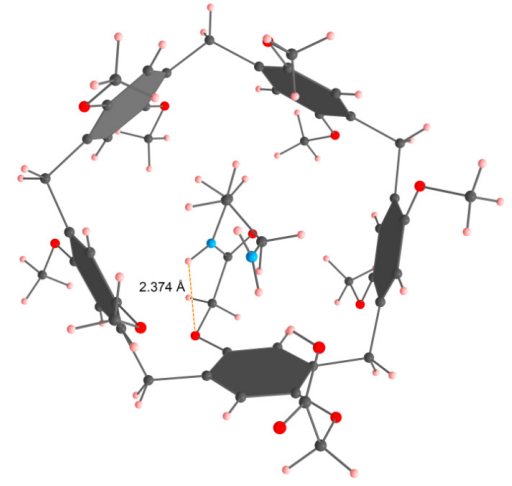

图 2 化合物 $\mathbf{8}$ 的单晶结构

Figure 2 Single crystal structure of compound 8

在分子内氢键作用的诱导下, 骨架上的侧链发生内翻, 使得侧链上的氨基位于柱 [5]芳烃孔穴内, 并且通过调 节烷基链的长度可以控制氨基的位置. 这类双官能团衍 生化的柱[5]芳烃为进一步构筑可进行多位点识别的主 体分子以及分子机器提供了很好的模块.

\section{3 实验部分}

\section{1 仪器与试剂}

${ }^{1} \mathrm{H}$ NMR 及 ${ }^{13} \mathrm{C}$ NMR 使用 Varain Mercury plus 400 型核磁共振仪测定 $(298 \mathrm{~K})$, 化学位移以氛代试剂残余 峰为参考; HR-MS 用 Bruker Micro TOF II 型质谱仪测 定.

\section{2 实验方法}

\subsection{1 化合物 $\mathbf{2}$ 的合成}

化合物 2 参考文献[13]方法合成, 并做了改进. 向 化合物 1 (3.00 g, $4.00 \mathrm{mmol})$ 的 $\mathrm{CH}_{2} \mathrm{Cl}_{2}(300 \mathrm{~mL})$ 溶液中 加入 $\left(\mathrm{NH}_{4}\right)_{2} \mathrm{Ce}\left(\mathrm{NO}_{3}\right)_{6}(4.38 \mathrm{~g}, 8.00 \mathrm{mmol})$ 和 $\mathrm{H}_{2} \mathrm{O}(3 \mathrm{~mL})$, 室温摚拌 $7 \mathrm{~min}$, 再加入 $\mathrm{H}_{2} \mathrm{O}$ 将固体溶解并分出有机相. 有机相分别用饱和食盐水洗涤、无水硫酸钠干燥. 过滤 得到的溶液减压浓缩至干得到固体, 接着将该固体悬浮 在乙酸乙酯中，搅拌并过滤以除去未反应的化合物 1 . 粗产物经柱层析 $[V$ (正己烷) $: V$ (乙酸乙酯 $)=30 ： 1]$ 分离 纯化, 得 $0.92 \mathrm{~g}$ 红色固体 2 , 产率 $31 \% .{ }^{1} \mathrm{H} \mathrm{NMR}\left(\mathrm{CDCl}_{3}\right.$, $400 \mathrm{MHz}) \delta: 6.84$ (s, 2H), 6.81 (s, 2H), 6.80 (s, 2H), 6.67 (s, 4H), 3.79 (s, 6H), 3.74 (s, 6H), 3.72 (d, J=4 Hz, 4H), $3.63(\mathrm{~s}, 6 \mathrm{H}), 3.59$ (s, 4H); ${ }^{13} \mathrm{C} \mathrm{NMR}\left(\mathrm{CDCl}_{3}, 100 \mathrm{MHz}\right) \delta$ : $188.6,151.0,150.7,150.6,146.4,133.3,129.2,128.2$, $127.8,123.4,114.1,113.7,113.6,55.9,55.8,55.6,55.3$, 29.3, 27.9.

\subsection{2 化合物 3 和 $\mathbf{4}$ 的合成 \\ 化合物 3 和 4 按照文献[14]方法合成.}

3: ${ }^{1} \mathrm{H}$ NMR $\left(\mathrm{CDCl}_{3}, 400 \mathrm{MHz}\right) \delta: 6.91(\mathrm{~s}, 2 \mathrm{H}), 6.85$ (s, 2H), 6.82 (s, 2H), 6.61 (s, 2H), 6.59 (s, 2H), 3.84 (s, 
6H), 3.77 (s, 12H), 3.74 (s, 6H), 3.69 (s, 10H).

4: ${ }^{1} \mathrm{H}$ NMR $\left(\mathrm{CDCl}_{3}, 400 \mathrm{MHz}\right) \delta: 6.85(\mathrm{~s}, 4 \mathrm{H}), 6.83$ (s, 2H), $6.78(\mathrm{~s}, 2 \mathrm{H}), 6.71(\mathrm{~s}, 2 \mathrm{H}), 4.55(\mathrm{~s}, 4 \mathrm{H}), 3.75(\mathrm{~s}$, $16 \mathrm{H}), 3.73(\mathrm{~s}, 6 \mathrm{H}), 3.70(\mathrm{~s}, 6 \mathrm{H}), 3.68(\mathrm{~s}, 6 \mathrm{H}), 3.13$ (q, J=8 $\mathrm{Hz}, 4 \mathrm{H}),-0.26$ (t, $J=8 \mathrm{~Hz}, 6 \mathrm{H})$.

\section{2 .3 化合物 $\mathbf{5}$ 的合成}

向化合物 4 (1.04 g, $1.15 \mathrm{mmol})$ 的四氢呋喃(THF)溶 液 $(20 \mathrm{~mL})$ 中逐滴滴加溶有 $\mathrm{NaOH}(0.46 \mathrm{mg}, 1.15 \mathrm{mmol})$ 的混合溶液 $\left(20 \mathrm{~mL}\right.$ THF, $2 \mathrm{~mL} \mathrm{H} \mathrm{H}_{2} \mathrm{O}, 2 \mathrm{~mL}$ EtOH), $40{ }^{\circ} \mathrm{C}$ 油浴下搅拌, 调节流速, 控制滴加时间为 $1 \mathrm{~h}$. 减压蒸馏 除去 THF, 乙酸乙酯溶解粗产物, 分别用稀盐酸、饱和 食盐水洗涤, 无水硫酸钠干燥. 粗产物经柱层析 $\left[V\left(\mathrm{CH}_{2} \mathrm{Cl}_{2}\right): V\left(\mathrm{CH}_{3} \mathrm{OH}\right)=10: 1\right]$ 分离出纯化, 得 $0.28 \mathrm{~g}$ 白色固体, 产率 30\%. ${ }^{1} \mathrm{H} \mathrm{NMR}\left(\mathrm{CDCl}_{3}, 400 \mathrm{MHz}\right) \delta: 6.89$ (s, 1H), $6.86(\mathrm{~s}, 1 \mathrm{H}), 6.84(\mathrm{~s}, 1 \mathrm{H}), 6.82(\mathrm{~s}, 1 \mathrm{H}), 6.81(\mathrm{~s}$, $1 \mathrm{H}), 6.77(\mathrm{~s}, 2 \mathrm{H}), 6.74(\mathrm{~s}, 1 \mathrm{H}), 6.61(\mathrm{~s}, 1 \mathrm{H}), 6.59(\mathrm{~s}, 1 \mathrm{H})$, $4.59(\mathrm{~s}, 2 \mathrm{H}), 4.53(\mathrm{~s}, 2 \mathrm{H}), 3.81 \sim 3.63(\mathrm{~m}, 34 \mathrm{H}), 2.45(\mathrm{q}$, $J=5.2 \mathrm{~Hz}, 2 \mathrm{H}),-1.08(\mathrm{t}, J=8 \mathrm{~Hz}, 3 \mathrm{H}) ;{ }^{13} \mathrm{C}$ NMR $\left(\mathrm{CDCl}_{3}, 100 \mathrm{MHz}\right) \delta: 173.2,168.9,151.0,150.9,150.6$, $150.4,150.1,149.9,149.8,147.8,129.5,128.7,128.6$, $128.5,128.4,128.3,127.8,126.8,115.7,114.9,114.2$, 113.9, 113.3, 112.9, 66.3, 64.9, 60.6, 55.9, 55.8, 55.7, 55.6, 55.3, 31.1, 29.9, 29.0, 28.9, 27.8, 11.1; HRMS (ESI-TOF) calcd for $\mathrm{C}_{49} \mathrm{H}_{53} \mathrm{O}_{14}[\mathrm{M}-\mathrm{H}]^{-}$865.3435, found 865.3411.

3.2 .4 化合物 6 的合成

向溶有化合物 5 (200 mg, $0.23 \mathrm{mmol})$ 的无水 THF (6 $\mathrm{mL}$ )中加入 $0.4 \mathrm{~mL}$ 的草酰氯, 反应 $3 \mathrm{~h}$ 后, 浓缩至干, 真 空干燥 $2 \mathrm{~h}, 5 \mathrm{~mL}$ THF 溶解, 依次加入 $66 \mu \mathrm{L}$ 无水三乙胺 和溶有相应烷基胺的 THF 溶液 $(0.46 \mathrm{mmol}, 5 \mathrm{~mL}$ 无水 $\mathrm{THF}$, 室温反应过夜, 浓缩至干, $\mathrm{CH}_{2} \mathrm{Cl}_{2}$ 溶解, 分别用 稀盐酸、饱和食盐水洗涤, 无水硫酸钠干燥. 粗产品经 柱层析 $\left[V\left(\mathrm{CH}_{2} \mathrm{Cl}_{2}\right): V\left(\mathrm{CH}_{3} \mathrm{OH}\right)=20: 1\right.$ 得白色固体.

6a: $160 \mathrm{mg}$, 产率 68\%, ${ }^{1} \mathrm{H} \mathrm{NMR}\left(\mathrm{CDCl}_{3}, 400 \mathrm{MHz}\right)$ $\delta: 6.97 \sim 6.77(\mathrm{~m}, 10 \mathrm{H}), 4.68 \sim 4.30(\mathrm{br}, 6 \mathrm{H}), 3.86 \sim 3.71$ (m, 34H), $3.01(\mathrm{br}, 1 \mathrm{H}), 1.60(\mathrm{~s}, 3 \mathrm{H}), 1.39(\mathrm{~s}, 9 \mathrm{H}), 0.30(\mathrm{br}$, 1H), 0.02 (br, 1H), -0.11 (br, 1H), -2.05 (br, 1H), -2.17 (br, $1 \mathrm{H}) ;{ }^{13} \mathrm{C}$ NMR $\left(\mathrm{CDCl}_{3}, 100 \mathrm{MHz}\right) \delta: 169.4$, $166.7,153.8,151.3,150.5,150.4,150.3,150.2,150.0$, $149.8,149.3,148.7,148.4,130.9,129.6,128.5,128.4$, $128.1,128.0,126.2,125.3,115.6,113.9,113.2,113.0$, $112.9,112.6,66.4,60.8,55.9,55.5,55.3,55.2,55.0,36.5$, 35.7, 31.5, 29.4, 28.7, 28.4, 27.2, 24.9, 14.1; HRMS (ESI-TOF) calcd for $\mathrm{C}_{57} \mathrm{H}_{71} \mathrm{~N}_{2} \mathrm{O}_{15}[\mathrm{M}+\mathrm{H}]^{+} 1023.4854$, found 1023.4849 .

6b: $167 \mathrm{mg}$, 产率 $70 \%,{ }^{1} \mathrm{H} \mathrm{NMR}\left(\mathrm{CDCl}_{3}, 400 \mathrm{MHz}\right)$ $\delta: 7.02 \sim 6.78(\mathrm{~m}, 10 \mathrm{H}), 4.79 \sim 4.53(\mathrm{~m}, 4 \mathrm{H}), 4.33 \sim 4.29$ (m, 2H), $3.92 \sim 3.73(\mathrm{~m}, 34 \mathrm{H}), 3.43(\mathrm{br}, 1 \mathrm{H}), 2.32(\mathrm{br}, 1 \mathrm{H})$, 1.45 (s, 9H), 1.35 (t, $J=6 \mathrm{~Hz}, 3 \mathrm{H}$ ), $1.18 \sim 1.13$ (br, $1 \mathrm{H}$ ), 0.92 (br, $1 \mathrm{H}), 0.81(\mathrm{br}, 1 \mathrm{H}),-1.87(\mathrm{br}, 1 \mathrm{H}),-2.00$ (br, $1 \mathrm{H}),-2.33$ (br, $2 \mathrm{H}) ;{ }^{13} \mathrm{C}$ NMR $\left(\mathrm{CDCl}_{3}, 100 \mathrm{MHz}\right) \delta$ : $169.1,166.8,154.6,151.0,150.3,150.2,150.0,148.6$, $148.1,130.7,129.6,128.8,128.5,128.3,128.0,127.8$, $127.7,126.7,114.6,114.4,113.1,113.0,112.8,112.7$, 112.6, 66.1, 61.1, 55.9, 55.7, 55.5, 55.4, 55.3, 40.2, 37.3, $30.7,29.3,28.7,28.4,27.9,23.3,22.2,14.2$; HRMS (ESI-TOF) calcd for $\mathrm{C}_{58} \mathrm{H}_{73} \mathrm{~N}_{2} \mathrm{O}_{15}[\mathrm{M}+\mathrm{H}]^{+}$1037.5011, found 1037.5005 .

6c: $160 \mathrm{mg}$, 产率 66\%, ${ }^{1} \mathrm{H}$ NMR $\left(\mathrm{CDCl}_{3}, 400 \mathrm{MHz}\right)$ $\delta: 7.19 \sim 6.76(\mathrm{~m}, 10 \mathrm{H}), 5.60(\mathrm{br}, 1 \mathrm{H}), 4.82 \sim 4.78(\mathrm{~m}, 1 \mathrm{H})$, $4.61(\mathrm{~s}, 2 \mathrm{H}), 4.55 \sim 4.51(\mathrm{~m}, 1 \mathrm{H}), 4.38 \sim 4.21(\mathrm{~m}, 2 \mathrm{H})$, $3.89 \sim 3.68(\mathrm{~m}, 34 \mathrm{H}), 3.05(\mathrm{br}, 1 \mathrm{H}), 2.07(\mathrm{br}, 1 \mathrm{H}), 1.43(\mathrm{~s}$, 9H), 1.35 (t, $J=8 \mathrm{~Hz}, 3 \mathrm{H}), 1.06$ (br, 1H), 0.91 (br, 1H), -0.30 (br, 1H), -0.48 (br, 1H), $-2.15(\mathrm{br}, 1 \mathrm{H}),-2.31$ (br, 3H); ${ }^{13} \mathrm{C}$ NMR $\left(\mathrm{CDCl}_{3}, 100 \mathrm{MHz}\right) \delta: 169.2,167.7$, $154.6,150.8,150.2,150.1,149.7,149.0,146.9,129.1$, $128.5,128.3,128.1,128.0,127.6,127.3,113.8,113.7$, $113.3,113.0,112.8,112.7,112.2,111.6,65.7,65.1,60.8$, $55.8,55.7,55.6,55.4,55.3,55.0,54.9,40.3,37.5,29.5$, $29.1,29.0,28.9,28.3,27.3,24.9,20.3,14.2$; HRMS (ESI-TOF) calcd for $\mathrm{C}_{59} \mathrm{H}_{75} \mathrm{~N}_{2} \mathrm{O}_{15}[\mathrm{M}+\mathrm{H}]^{+}$1051.5167, found 1051.5162 .

\section{2 .5 化合物 7 的制备}

向化合物 $6(0.16 \mathrm{mmol})$ 的 $\mathrm{CH}_{2} \mathrm{Cl}_{2}(3 \mathrm{~mL})$ 溶液中加 入三氟乙酸 $(1 \mathrm{~mL})$, 室温搅拌 $10 \mathrm{~h}$ 后, 加 $\mathrm{CH}_{2} \mathrm{Cl}_{2}$ 稀释反 应液, 分别用饱和碳酸氢钠溶液、饱和食盐水洗涤, 无 水硫酸钠干燥, 浓缩至干得淡黄色固体.

7a: $132 \mathrm{mg}$, 产率 90\%, ${ }^{1} \mathrm{H}$ NMR $\left(\mathrm{CDCl}_{3}, 500 \mathrm{MHz}\right)$ $\delta: 7.05 \sim 6.75(\mathrm{~m}, 10 \mathrm{H}), 5.36(\mathrm{~s}, 2 \mathrm{H}), 4.66(\mathrm{~s}, 2 \mathrm{H}), 4.56(\mathrm{~s}$, $2 \mathrm{H}), 4.24(\mathrm{~d}, J=10 \mathrm{~Hz}, 2 \mathrm{H}), 3.81 \sim 3.72(\mathrm{~m}, 34 \mathrm{H}), 2.58$ (br, 1H), $1.27(\mathrm{t}, J=5 \mathrm{~Hz}, 3 \mathrm{H}), 1.39(\mathrm{~s}, 9 \mathrm{H}),-0.85(\mathrm{~s}$, $2 \mathrm{H}),-1.03(\mathrm{br}, 2 \mathrm{H}) ;{ }^{13} \mathrm{C}$ NMR $\left(\mathrm{CDCl}_{3}, 100 \mathrm{MHz}\right) \delta$ : $169.3,167.1,151.2,150.4,150.2,150.0,149.5,148.8$, $147.9,130.0,129.5,128.6,128.5,128.4,128.3,128.2$, $127.0,126.7,115.3,113.8,113.6,113.5,113.3,113.1$, $112.8,112.7,112.3,66.1,65.9,61.1,55.9,55.7,55.6,55.5$, 55.4, 36.2, 35.2, 30.6, 29.0, 28.9, 28.3, 28.1, 14.0; HRMS (ESI-TOF) calcd for $\mathrm{C}_{52} \mathrm{H}_{63} \mathrm{~N}_{2} \mathrm{O}_{13}[\mathrm{M}+\mathrm{H}]^{+}$923.4330, found 923.4325 .

7b: $138 \mathrm{mg}$, 产率 $92 \%,{ }^{1} \mathrm{H} \mathrm{NMR}\left(\mathrm{CDCl}_{3}, 400 \mathrm{MHz}\right)$ $\delta: 6.97 \sim 6.80(\mathrm{~m}, 10 \mathrm{H}), 4.80(\mathrm{~s}, 2 \mathrm{H}), 4.55(\mathrm{~d}, J=16 \mathrm{~Hz}$, 
2H), 4.28 (s, 2H), 3.97 3.74 (m, 34H), 2.27 (br, 1H), 1.59 (br, $1 \mathrm{H}), 1.33$ (t, $J=6 \mathrm{~Hz}, 3 \mathrm{H}), 0.48 \sim 0.40$ (br, 2H), 1.72 (br, 1H), -1.95 (br, 1H), -2.39 (br, $2 \mathrm{H}) ;{ }^{13} \mathrm{C} \mathrm{NMR}$ $\left(\mathrm{CDCl}_{3}, 100 \mathrm{MHz}\right) \delta: 169.0,167.1,150.8,150.4,150.2$, $150.0,149.9,148.5,148.1,130.4,129.6,128.6,128.1$, $128.0,126.7,126.6,114.7,113.6,113.2,113.0,112.9$, $112.7,112.6,65.9,61.4,55.5,55.4,55.1,41.0,40.8,37.8$, 30.6, 29.7, 28.5, 27.7, 22.1, 14.1; HRMS (ESI-TOF) calcd for $\mathrm{C}_{53} \mathrm{H}_{65} \mathrm{~N}_{2} \mathrm{O}_{13}[\mathrm{M}+\mathrm{H}]^{+}$937.4487, found 937.4542.

7c: $134 \mathrm{mg}$, 产率 $88 \%,{ }^{1} \mathrm{H} \mathrm{NMR}\left(\mathrm{CDCl}_{3}, 400 \mathrm{MHz}\right)$ $\delta: 7.08 \sim 6.72(\mathrm{~m}, 10 \mathrm{H}), 5.64(\mathrm{br}, 2 \mathrm{H}), 4.65 \sim 4.59(\mathrm{~m}, 4 \mathrm{H})$, 4.31 (br, $2 \mathrm{H}), 3.83 \sim 3.70(\mathrm{~m}, 34 \mathrm{H}), 3.11(\mathrm{br}, 1 \mathrm{H}), 1.87$ (br, 1H), 1.32 (t, $J=6 \mathrm{~Hz}, 3 \mathrm{H}), 0.94$ (br, 2H), -0.37 (br, 2H), -2.06 (br, 2H), -2.23 (br, $2 \mathrm{H}) ;{ }^{13} \mathrm{C}$ NMR $\left(\mathrm{CDCl}_{3}, 100\right.$ MHz) $\delta: 169.2$, 167.4, 150.7, 150.6, 150.4, 150.3, 150.1, $148.8,147.7,129.7,129.6,128.7,128.5,128.4,128.2$, $127.6,127.5,127.3,114.5,114.0,113.5,113.4,113.3$, $112.8,112.4,112.2,65.8,61.5,56.4,56.0,55.8,55.7,55.4$, 55.2, 40.9, 37.0, 30.0, 29.2, 28.9, 28.7, 28.2, 27.6, 19.3; HRMS (ESI-TOF) calcd for $\mathrm{C}_{54} \mathrm{H}_{67} \mathrm{~N}_{2} \mathrm{O}_{13}[\mathrm{M}+\mathrm{H}]^{+}$ 951.4643, found 951.4638 .

\section{2 .5 化合物 8 的制备}

向化合物 $7 \mathbf{a}(132 \mathrm{mg}, 0.14 \mathrm{mmol})$ 的 $\mathrm{CH}_{2} \mathrm{Cl}_{2}(3 \mathrm{~mL})$ 溶液中加入溶有 $\mathrm{NaOH}(11 \mathrm{mg})$ 的 $\mathrm{THF}$ 和 $\mathrm{H}_{2} \mathrm{O}(V: V=$ $3: 1)$ 的混合溶液, 室温搅拌 $10 \mathrm{~h}$ 后, 稀盐酸酸化, 加 $\mathrm{CH}_{2} \mathrm{Cl}_{2}$ 溶解, 分别用饱和碳酸氢钠溶液、饱和食盐水洗 涤, 无水硫酸钠干燥, 浓缩至干得 $114 \mathrm{mg}$ 淡黄色固体, 产率 91\%. ${ }^{1} \mathrm{H} \mathrm{NMR}\left(\mathrm{CDCl}_{3}, 400 \mathrm{MHz}\right) \delta: 7.15 \sim 6.73(\mathrm{~m}$, $10 \mathrm{H}), 5.88$ (br, 2H), 4.71 (s, 2H), 4.44 (q, $J=16 \mathrm{~Hz}, 2 \mathrm{H})$, $3.91 \sim 3.69$ (m, 34H), 1.55 (br, 1H), 0.09 (br, 1H), -0.15 (br, 2H), -1.47 (br, $1 \mathrm{H}),-1.63$ (br, $1 \mathrm{H}) ;{ }^{13} \mathrm{C} \mathrm{NMR}$ $\left(\mathrm{CDCl}_{3}, 100 \mathrm{MHz}\right) \delta: 176.1,167.0,151.3,150.9,150.5$, $150.3,150.2$, 149.9, 148.7, 147.9, 131.0, 130.1, 129.4, $129.1,129.0,128.8,128.0,126.0,114.6,114.1,114.0$, $112.8,112.7,112.6,66.4,66.0,57.1,56.6,56.5,56.2,56.0$, 55.8, 55.1, 35.1, 34.2, 32.6, 29.8, 28.8, 27.7, 26.7, 24.7. HRMS (ESI-TOF) calcd for $\mathrm{C}_{50} \mathrm{H}_{59} \mathrm{~N}_{2} \mathrm{O}_{13}[\mathrm{M}+\mathrm{H}]{ }^{+}$ 895.4017, found 895.4012.

\section{3 晶体结构}

化合物 8, CCDC 1474985, 分子式: $\mathrm{C}_{51} \mathrm{H}_{60} \mathrm{Cl}_{2} \mathrm{~N}_{2} \mathrm{O}_{14}$; 相对分子质量 $\left(M_{\mathrm{r}}\right)$ : 995.91 ; 晶系: $P-1$; 晶胞参数: $a=$ 11.571(3) $\AA, b=12.215(3) \AA, c=19.497(5) \AA, \alpha=$ $85.936(4)^{\circ}, \beta=82.733(4)^{\circ}, \gamma=68.235(4)^{\circ}$; $\quad$ 体 积 : $2537.8(11) \AA^{3}$; 密度实验值 $\left(D_{\mathrm{m}}\right): 1.303 \mathrm{~g} / \mathrm{cm}^{3}$; 计算值 $\left(D_{\mathrm{c}}\right): 1.303 \mathrm{~g} / \mathrm{cm}^{3}$; 晶胞中分子数 $(\mathrm{Z}): 2 ; F(000): 1052$.
辅助材料(Supporting Information) 化合物 $2 \sim 8$ 的核 磁共振氢谱、碳谱以及高分辨质谱. 这些材料可以免费 从本刊网站(http://sioc-journal.cn/)上下载.

\section{References}

[1] Ogoshi, T.; Kanai, S.; Fujinami, T.-A.; Nakamoto, Y. J. Am. Chem. Soc. 2008, 130, 5022.

[2] (a) Ogoshi, T.; Yamagishi, T. A. Eur. J. Org. Chem. 2013, 15, 2961. (b) Xue, M.; Yang, Y.; Chi, X.; Zhang, Z.; Huang, F. Acc. Chem. Res. 2012, 45, 1294.

(c) Han, C.; Yu, G.; Zheng, B.; Huang, F. Org. Lett. 2012, 14, 1712. (d) Cragg, P.-J.; Sharma, K. Chem. Soc. Rev. 2012, 41, 597. (e) Han, C.; Yu, G.; Zheng, B.; Huang, F. Org. Lett. 2012, 14, 1712. (f) Li, C.; Chen, S.; Li, J.; Han, K.; Xu, M.; Hu, B.; Yu, Y.; Jia, X. Chem. Commun. 2011, 47, 11294.

(g) Li, C.; Xu, Q.; Li, J.; Yao, F.; Jia, X. Org. Biomol. Chem. 2010, $8,1568$.

(h) Cao, D.; Kou, Y.; Liang, J.; Chen, Z.; Wang, L.; Meier, H. Angew. Chem., Int. Ed. 2009, 48, 9721.

[3] (a) Chen, H.; Fan, J.; Hu, X.; Ma, J.; Wang, S.; Li, J.; Yu, Y.; Jia, X.; Li, C. Chem. Sci. 2015, 6, 197.

(b) Strutt, N. L.; Zhang, H. C.; Schneebeli, S. T.; Stoddart, J. F. Acc. Chem. Res. 2014, 47, 2631

(c) Yue, S. Y.; Zhou, Y. J.; Yao, Y.; Xue, M. Acta Chim. Sinica 2014, 72, 1053 (in Chinese).

(岳诗雨, 周玉娟, 姚勇, 薛敏, 化学学报, 2014, 72, 1053.)

(d) Han, C.; Zhang, Z.; Chi, X.; Zhang, M.; Yu, G.; Huang, F. Acta Chim. Sinica 2012, 70, 1775 (in Chinese).

(韩成友, 张子彬, 池小东, 张明明, 喻国灿, 黄飞鹤, 化学学报, 2012, 70, 1775.)

(e) Zhang, H.-C.; Liu, Z.-N.; Xin, F.-F.; Hao, A.-Y. Chin. J. Org. Chem. 2012, 32, 219 (in Chinese). (张华承，刘召娜，辛飞飞, 郝爱友，有机化学, 2012, 32, 219.)

[4] (a) Wang, S.; Wang, Y.; Chen, Z.; Lin. Y.; Weng, L.; Han, K.; Li, J.; Jia, X.; Li, C. Chem. Commun. 2015, 51, 3434.

(b) Li, C. Chem. Commun. 2014, 50, 12420.

(c) Hu, X.-Y.; Wu, X.; Wang, S.; Chen, D.; Xia, W.; Lin, C.; Pan, Y.; Wang, L. Polym. Chem. 2013, 4, 4292.

(d) Ogoshi, T.; Kayama, H.; Yamafuji, D.; Aoki, T.; Yamagishi, T. Chem. Sci. 2012, 3, 3221.

(e) Guan, Y.; Ni, M.; Hu, X.; Xiao, T.; Xiong, S.; Lin, C.; Wang, L. Chem. Commun. 2012, 48, 8529.

(f) Zhang, Z.; Han, C. Yu, G.; Huang, F. Chem. Sci. 2012, 3, 3026. (g) Zhang, Z.; Luo, Y.; Chen, J.; Dong, S.; Yu, Y.; Ma, Z.; Huang, F. Angew. Chem., Int. Ed. 2011, 50, 1397.

[5] (a) Dong, S.; Yuan, J.; Huang, F. Chem. Sci. 2014, 5. 247.

(b) Xia, B.; Xue, M. Chem. Commun. 2014, 50, 1021.

(c) Han, K.; Zhang, Y.; Li, J.; Yu, Y.; Jia, X.; Li, C. Eur. J. Org. Chem. 2013, 15, 2057.

(d) Zhang, Z.; Han, C.; Yu, G.; Huang, F. Chem. Sci. 2012, 3, 3026. (e) Strutt, N. L.; Forgan, R. S.; Spruell, J. M.; Botros, Y. Y.; Stoddart, J. F. J. Am. Chem. Soc. 2011, 133, 5668.

[6] Si, W.; Xin, P.-Y.; Li, Z.-T.; Hou, J.-L. Acc. Chem. Res. 2015, 48, 1612.

[7] (a) Cao, Y.; Hu, X.-Y.; Li Y.; Zou, X.; Xiong, S.; Lin, C.; Wang, L. J. Am. Chem. Soc. 2014, 136, 10762.

(b) Zhou, J.; Chen, M.; Diao, G. Chem. Commun. 2014, 50, 11954.

(c) Chang, Y.; Yang, K.; Wei, P.; Huang, S.; Pei, Y.; Zhao, W.; Pei, Z. Angew. Chem., Int. Ed. 2014, 53, 13126.

(d) Mang, M.-F.; Xing, P.-Y.; Li, S.-Y.; Chu, X.-X.; Wang, B.; Hao, 
A.-Y. Prog. Chem. 2014, 26, 1317 (in Chinese).

(马明放, 邢鹏遥, 李尚鹏, 初晓晓, 王波, 郝爱友, 化学进展, 2014, 26, 1317.)

(e) Duan, Q.; Cao, Y.; Li, Y.; Hu, X.; Xiao, T.; Lin, C.; Pan, Y.; Wang, L. J. Am. Chem. Soc. 2013, 135, 10542.

(f) Yao, Y.; Xue, M.; Chen, J.; Zhang, M.; Huang, F. J. Am. Chem. Soc. 2012, 134, 15712.

(g) Yu, G.; Zhou, X.; Zhang, Z.; Han, C.; Mao, Z.; Gao, C.; Huang, F. J. Am. Chem. Soc. 2012, 134, 19489.

[8] (a) Zhang, C.-W.; Chen, L.-J.; Yang, H.-B. Chin. J. Chem. 2015, 33, 319.

(b) Wang, W.; Chen, L.-J. Wang, X.-Q.; Sun, B.; Li, X.; Zhang, Y. Shi, J.; Yu, Y.; Zhang, L.; Liu, M.; Yang, H.-B. Proc. Natl. Acad. Sci. U. S. A. 2015, 112, 5597.

(c) Tan, L. L.; Li, H.; Zhou, Y.; Zhang, Y.; Feng, X.; Wang, B.; Yang, Y.-W. Small 2015, 11, 3807.

(d) Tan, L.-L.; Li, H.; Tao, Y.; Zhang, S. X.-A.; Wang, B.; Yang, Y.-W. Adv. Mater. 2014, 26, 7027.

(e) Li, H.; Chen, D.-X.; Sun, Y.-L.; Zheng, Y. B.; Tan, L. L.; Weiss, P. S.; Yang, Y.-W. J. Am. Chem. Soc. 2013, 135, 1570.

(f) Zhang, H.; Gruner, G.; Zhao, Y. J. Mater. Chem. B 2013, 1, 2542.

(g) Zhang, H.; Ma, X.; Nguyen, K. T.; Zhao, Y. ACS Nano. 2013, 7, 7853. (h) Gao, L.; Zheng, B.; Yao, Y.; Huang, F. Soft Matter 2013, 9, 7314.

[9] (a) Yao, Y.; Xue, M.; Chi, X.; Ma, Y.; He, J.; Abliz, Z.; Huang, F. Chem. Commun. 2012, 48, 6505.

(b) Hu, X.-B.; Chen, L.; Si, W.; Yu, Y.; Hou, J.-L. Chem. Commun. 2011, 47, 4694.

(c) Ogoshi, T.; Hashizume, M.; Yamagishi, T.; Nakamoto, Y. Chem. Commun. 2010, 46, 3708.

(d) Ogoshi, T.; Umeda, K.; Yamagishi, T.; Nakamoto, Y. Chem. Commun. 2009, 45, 4874.

[10] (a) Wei, P.; Yan, X.; Li, J.; Ma, Y.; Huang, F. Chem. Commun. 2013, 49, 1070.

(b) Yu, G.; Zhang, Z.; Han, C.; Xue. M.; Zhou, Q.; Huang, F. Chem. Commun. 2012, 48, 2958.

[11] Ogoshi, T.; Demachi, K.; Kitajima, K.; Yamagishi, T. Chem. Commun. 2011, 47, 7164.

[12] Ogoshi, T. Pillararenes, The Royal Society of Chemistry, Cambridge, UK, 2016.

[13] Xie, C.-D.; Hu, W.-B.; Hu, W.-J.; Liu, Y.-A.; Huo, J.-C.; Li, J.-S. Jiang, B.; Wen, K. Chin. J. Chem. 2015, 33, 379.

[14] Ogoshi, T.; Yamafuji, D.; Kotera, D.; Aoki, T.; Fujinami, S.; Yamagishi, T. J. Org. Chem. 2012, 77, 11146.

[15] Chen, L.; Li, Z.; Chen, Z.; Hou, J.-L. Org. Biomol. Chem. 2013, 11, 248.

(Zhao, C.) 